\title{
Dynamic Modeling and Simulation of Marine Satellite Tracking Antenna Using Lagrange Method
}

\author{
Yunlong Wang, Mohsen Soltani and Dil Muhammad Akbar Hussain \\ Department of Energy Technology \\ Aalborg University \\ Esbjerg 6700, Denmark \\ Email: way@et.aau.dk
}

\begin{abstract}
Marine Satellite Tracking Antenna (MSTA) is a necessary device in ships for receiving satellite signals when they are sailing on the sea. This paper presents a simple methodology to obtain the dynamic equations of MSTA through Lagrange method, which is fundamental in design of modelbased controllers. The detailed derivation procedure of using basis vectors of coordinate system, is presented. Moreover, modern softwares, such as Maple and Matlab, are used for simulating the dynamic model of MSTA, whose results are compared to that from SimMechanics for validation. The proposed methods and tools can be easily applied on other mechanical systems.
\end{abstract}

Keywords-Marine Satellite Tracking Antenna; Lagrange method; SimMechanics

\section{INTRODUCTION}

Marine Satellite Tracking Antenna (MSTA) is an important device for ships to continuously communicate with geosynchronous satellite. With the use of attitude control system, MSTA can keep tracking a certain geosynchronous satellite, whatever the ship sways due to the ocean waves. The prerequisite of designing model-based controllers, such as Model Predictive Controller (MPC), is deriving the dynamic model of MSTA firstly.

The previous works about modelling MSTA can be found in [1], [2], [3], where the modeling of MSTA is divided into three parts, that is, a model for satellite tracking, a model for antenna dynamics, and a model of ship motion. However, these works mainly focus on the kinematics of MSTA. In [4], only the linear model of MSTA is stated for controller design, no kinematic or dynamic model is mentioned. In [5], [6], the Lagrange method is used to obtain the dynamics of MSTA, but it is assumed that the position of mass center of each part of MSTA will not be changed by the rotation of each part, which results in the kinetic energy of mass center to be zero. Lagrange method is one of the two classic methods for obtaining dynamics of a mechanical system, another is Newton-Euler method. Many researching works can be found using Lagrange method for system dynamic modeling [7], [8], [9]. The shortcomings of these works are that they mainly focus on equation derivation, and almost no modern modeling and simulation tools are introduced to solve complicated equations. In fact, when dealing with real mechanical systems, the scale of complexity of mathematical equations usually exceed the limit that we can handle manually.

The contributions of this paper are: (1) Detailed derivation procedure using Lagrange method for obtaining dynamics of 3-DOF MSTA is presented, in which basis vectors of coordinate system are applied that are less complicated than using rotational matrix directly and can be used to deal with more complicated mechanical systems. (2) Modern simulation tools, such as Maple, Matlab and SimMechanics toolbox, are introduced to handle complicated dynamic system and to validate the dynamic model of MSTA.

This paper is organized as follows: In Section II, the configuration and reference frames of MSTA is introduced. The procedure of deriving dynamic model of MSTA is stated in detail in Section III. In Section IV, the derived model is simulated using Matlab, whose results are validated through comparing with that from SimMechanics. Finally, Section V gives the conclusion.

\section{Mechanical Configuration And RefEREnCE FRAMES}

The configuration and reference frames of the MSTA are introduced firstly for derivation of dynamics equations in the next section.

The MSTA consists of four parts, that is, base part, yaw part, roll part and pitch part. Correspondingly, four reference frames are defined:

1) Reference frame of base part, defined by $\left(x_{\text {base }}, y_{\text {base }}, z_{\text {base }}\right)$, with origin $o_{\text {base }}$, as shown in Figure 1(a). $\boldsymbol{i}_{\text {base }}, \boldsymbol{j}_{\text {base }}$, and $\boldsymbol{k}_{\text {base }}$ are mutually perpendicular unit vectors.

2) Reference frame of yaw part, defined by $\left(x_{\text {yaw }}, y_{\text {yaw }}, z_{\text {yaw }}\right)$, with origin oyaw, as shown in Figure $1(\mathrm{~b}) . \boldsymbol{i}_{y a w}, \boldsymbol{j}_{y a w}$, and $\boldsymbol{k}_{y a w}$ are mutually perpendicular unit vectors.

3) Reference frame of roll part, defined by $\left(x_{\text {roll }}, y_{\text {roll }}, z_{\text {roll }}\right)$, with origin $o_{\text {roll }}$, as shown in Figure $1(\mathrm{c}) . \boldsymbol{i}_{\text {roll }}, \boldsymbol{j}_{\text {roll }}$, and $\boldsymbol{k}_{\text {roll }}$ are mutually perpendicular unit vectors. 
4) Reference frame of pitch part, defined by $\left(x_{\text {pitch }}, y_{\text {pitch }}, z_{\text {pitch }}\right)$, with origin $o_{y a w}$, as shown in Figure $1(\mathrm{~d}) . \boldsymbol{i}_{\text {pitch }}, \boldsymbol{j}_{\text {pitch }}$, and $\boldsymbol{k}_{\text {pitch }}$ are mutually perpendicular unit vectors.

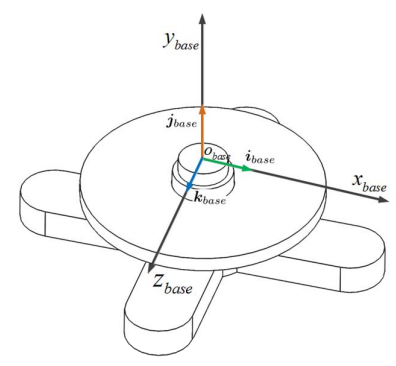

(a)

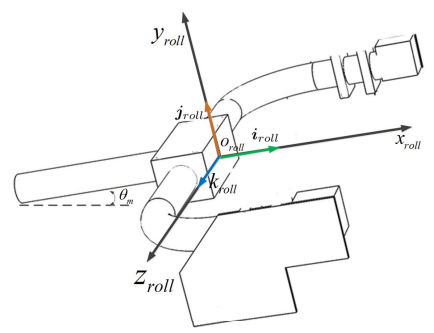

(c)

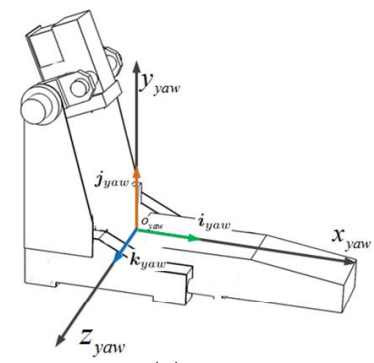

(b)

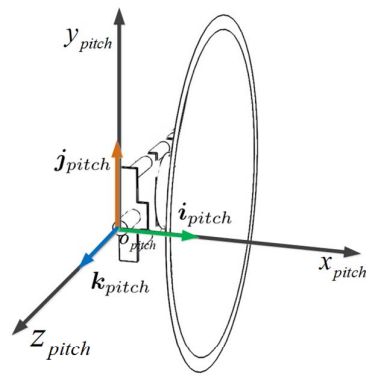

(d)
Figure 1. (a) Reference frame of base part. (b) Reference frame of yaw part. (c) Reference frame of roll part. (b) Reference frame of pitch part. Detailed explanation can be found in the patent [10].

There are three rotational axes in the MSTA :

1) azimuth axis, which is coincide with $\boldsymbol{y}_{\text {base }}$ in Figure $1(\mathrm{a})$.

2) cross-elevation axis, which is coincide with $\boldsymbol{x}_{\text {roll }}$ in Figure 1(c).

3) elevation axis, which is coincide with $z_{p i t c h}$ in Figure $1(d)$.

The three rotation angles corresponding to the above three rotation axes are

1) $\psi$ is the rotation angle of yaw frame around azimuth axis.

2) $\phi$ is the rotation angle of roll frame around crosselevation axis.

3) $\theta$ is the rotation angle of pitch frame around elevation axis.

$m_{y}, m_{r}$, and $m_{p}$ are the masses of yaw part, roll part, and pitch part respectively. $\theta_{m}$ is the mount angle of roll part, which is indicated in Figure 1(c). $\boldsymbol{I}_{y}, \boldsymbol{I}_{r}, \boldsymbol{I}_{p} \in R^{3 \times 3}$ are moments of inertia of yaw part, roll part, and pitch part respectively and $\boldsymbol{I}_{y}=\left(\boldsymbol{I}_{y}\right)^{T}, \boldsymbol{I}_{r}=\left(\boldsymbol{I}_{r}\right)^{T}, \boldsymbol{I}_{p}=\left(\boldsymbol{I}_{p}\right)^{T}$.

The following distance vectors are used to indicate the position relationships between different mass center:

1) $\boldsymbol{d}_{y m c}^{y}=\left[d x_{y m c}, d y_{y m c}, d z_{y m c}\right]^{T}$, is the distance vector from oyaw to mass center of yaw part, expressed in yaw frame.

2) $\boldsymbol{d}_{r m c}^{r}=\left[d x_{r m c}, d y_{r m c}, d z_{r m c}\right]^{T}$, is the distance vector from $o_{\text {roll }}$ to mass center of roll part, expressed in roll frame.

3) $\boldsymbol{d}_{p m c}^{p}=\left[d x_{p m c}, d y_{p m c}, d z_{p m c}\right]^{T}$, is the distance vector from $o_{\text {pitch }}$ to mass center of pitch part, expressed in pitch frame.

4) $\boldsymbol{d}_{b y}^{b}=\left[d x_{b y}, d y_{b y}, d z_{b y}\right]^{T}$, is the vector from origin of base frame, $o_{b}$, to origin of yaw frame, $o_{y}$, expressed in base frame.

5) $\boldsymbol{d}_{y r}^{y}=\left[d x_{y r}, d y_{y r}, d z_{y r}\right]^{T}$, is the vector from origin of yaw frame, $o_{y}$, to origin of roll frame, $o_{r}$, expressed in yaw frame.

6) $\boldsymbol{d}_{r p}^{r}=\left[d x_{r p}, d y_{r p}, d z_{r p}\right]^{T}$, is the vector from origin of roll frame, $o_{r}$, to origin of pitch frame, $o_{p}$, expressed in roll frame.

The damping coefficients of rotation around azimuth axis, cross-elevation axis, and elevation axis are $C_{d y}, C_{d r}$, and $C_{d p}$ respectively.

\section{Dynamic Equations Derivation}

The dynamic equations of MSTA are derived in this section using Lagrange method. There are three rotation parts in the MSTA and each part will be analysed separately, which are then combined together to obtain the whole dynamic model of MSTA. Instead of using rotation matrix directly, the unit vectors of reference frame are used in derivation, which are powerful enough for handling complicated mechanical system.

The yaw part is analysed firstly, then the roll part, and finally the pitch part. For each part, the kinetic energy is calculated firstly followed by the potential energy.

\section{A. Yaw part}

\section{1) Kinetic energy of mass center:}

The relationship between yaw frame and base frame is expressed as

$$
\begin{aligned}
\boldsymbol{i}_{\text {yaw }} & =\cos (\psi) \cdot \boldsymbol{i}_{\text {base }}-\sin (\psi) \cdot \boldsymbol{k}_{\text {base }} \\
\boldsymbol{j}_{\text {yaw }} & =\boldsymbol{j}_{\text {base }} \\
\boldsymbol{k}_{\text {yaw }} & =\sin (\psi) \cdot \boldsymbol{i}_{\text {base }}+\cos (\psi) \cdot \boldsymbol{k}_{\text {base }} .
\end{aligned}
$$

From (1) to (3), the rotational matrix from yaw frame to base frame is obtained

$$
\boldsymbol{R}_{y}^{b}=\left[\begin{array}{ccc}
\cos (\psi) & 0 & \sin (\psi) \\
0 & 1 & 0 \\
-\sin (\psi) & 0 & \cos (\psi)
\end{array}\right] .
$$

The position vector from $o_{b}$ to mass center of yaw part, expressed in base frame, is

$$
\boldsymbol{d}_{o y c}^{b}=\boldsymbol{d}_{b y}^{b}+\boldsymbol{d}_{y m c}^{b}
$$

where $\boldsymbol{d}_{y m c}^{b}=\boldsymbol{R}_{y}^{b} \cdot \boldsymbol{d}_{y m c}^{y}$, is the position vector from $o_{y a w}$ to mass center of yaw part, expressed in base frame. 
The velocity of mass center of yaw part expressed in base frame is

$$
\boldsymbol{v}_{y m c}^{b}=\frac{d\left(\boldsymbol{d}_{\text {oyc }}^{b}\right)}{d t}
$$

The kinetic energy of mass center of yaw part is

$$
E_{k y m}=\frac{1}{2} \cdot m_{y} \cdot\left\|\boldsymbol{v}_{y m c}^{b}\right\|_{2}^{2} .
$$

\section{2) Kinetic energy of moment of inertia:}

The angular velocity of yaw part is expressed in yaw frame as

$$
\boldsymbol{w}_{y}^{y}=\dot{\psi} \cdot \boldsymbol{j}_{\text {yaw }} .
$$

The kinetic energy from moment of inertia is

$$
E_{k y I}=\frac{1}{2} \cdot\left(\boldsymbol{w}_{y}^{y}\right)^{T} \cdot \boldsymbol{I}_{y} \cdot\left(\boldsymbol{w}_{y}^{y}\right) .
$$

\section{3) Total kinetic energy:}

The total kinetic energy of yaw part is the sum of kinetic energy from mass center and moment of inertia, and is expressed as

$$
E_{k y}=E_{k y m}+E_{k y I} .
$$

\section{4) Potential energy:}

The potential energy of yaw part is

$$
E_{p y}=0,
$$

as there is no displacement in the direction of gravity.

\section{B. Roll part}

1) Kinetic energy of mass center:

The relationship between roll frame and yaw frame is expressed as

$$
\begin{aligned}
\boldsymbol{i}_{\text {roll }}= & \cos \left(\theta_{m}\right) \cdot \boldsymbol{i}_{\text {yaw }}+\sin \left(\theta_{m}\right) \cdot \boldsymbol{j}_{\text {yaw }} \\
\boldsymbol{j}_{\text {roll }}= & \left(-\sin \left(\theta_{m}\right) \cdot \boldsymbol{i}_{\text {yaw }}+\cos \left(\theta_{m}\right) \cdot \boldsymbol{j}_{\text {yaw }}\right) \cdot \cos (\phi) \\
& +\sin (\phi) \cdot \boldsymbol{k}_{\text {yaw }} \\
\boldsymbol{k}_{\text {roll }}= & -\left(-\sin \left(\theta_{m}\right) \cdot \boldsymbol{i}_{\text {yaw }}+\cos \left(\theta_{m}\right) \cdot \boldsymbol{j}_{\text {yaw }}\right) \cdot \sin (\phi) \\
& +\cos (\phi) \cdot \boldsymbol{k}_{\text {yaw }} .
\end{aligned}
$$

From (13) to (14), the rotational matrix from roll frame to yaw frame is obtained

$\boldsymbol{R}_{r}^{y}=\left[\begin{array}{ccc}\cos \left(\theta_{m}\right) & -\sin \left(\theta_{m}\right) \cdot \cos (\phi) & \sin \left(\theta_{m}\right) \cdot \sin (\phi) \\ \sin \left(\theta_{m}\right) & \cos \left(\theta_{m}\right) \cdot \cos (\phi) & -\cos \left(\theta_{m}\right) \cdot \sin (\phi) \\ 0 & \sin (\phi) & \cos (\phi)\end{array}\right.$

The position vector from $o_{b}$ to mass center of roll part, expressed in base frame, is

$$
\boldsymbol{d}_{o r c}^{b}=\boldsymbol{d}_{b y}^{b}+\boldsymbol{d}_{y r}^{b}+\boldsymbol{d}_{r m c}^{b}
$$

where $\boldsymbol{d}_{y r}^{b}$ is the position vector from $o_{y a w}$ to $o_{\text {roll }}$, expressed in base frame, and $\boldsymbol{d}_{r m c}^{b}$ is the position vector from $o_{\text {roll }}$ to mass center of roll part, expressed in base frame. $\boldsymbol{d}_{y r}^{b}$ and $\boldsymbol{d}_{r m c}^{b}$ are obtained by

$$
\begin{aligned}
\boldsymbol{d}_{y r}^{b} & =\boldsymbol{R}_{y}^{b} \cdot \boldsymbol{d}_{y r}^{y} \\
\boldsymbol{d}_{r m c}^{b} & =\boldsymbol{R}_{y}^{b} \cdot \boldsymbol{R}_{r}^{y} \cdot \boldsymbol{d}_{r m c}^{r} .
\end{aligned}
$$

The velocity of mas center of roll part in base frame is

$$
\boldsymbol{v}_{o r c}^{b}=\frac{d\left(\boldsymbol{d}_{o r c}^{b}\right)}{d t} .
$$

The kinetic energy of mass center of roll part is

$$
E_{k r m}=\frac{1}{2} \cdot m_{r} \cdot\left\|\boldsymbol{v}_{o r c}^{b}\right\|_{2}^{2} .
$$

2) Kinetic energy of moment of inertia:

The angular velocity of roll part in roll frame is

$$
\boldsymbol{w}_{r}^{r}=\dot{\psi} \cdot \boldsymbol{j}_{\text {raw }}+\dot{\phi} \cdot \boldsymbol{i}_{\text {roll }} .
$$

Decompose $\boldsymbol{j}_{\text {raw }}$ into roll frame

$$
\begin{aligned}
\boldsymbol{j}_{\text {yaw }}= & \sin \left(\theta_{m}\right) \cdot \boldsymbol{i}_{\text {roll }} \\
& +\cos \left(\theta_{m}\right) \cos (\phi) \cdot \boldsymbol{j}_{\text {roll }} \\
& -\cos \left(\theta_{m}\right) \cdot \sin (\phi) \cdot \boldsymbol{k}_{\text {roll }} .
\end{aligned}
$$

Substitute (21) into (42),

$$
\begin{aligned}
\boldsymbol{w}_{r}^{r}= & \dot{\psi} \cdot \boldsymbol{j}_{\text {raw }}+\dot{\phi} \cdot \boldsymbol{i}_{\text {roll }} \\
= & \dot{\psi} \cdot\left(\sin \left(\theta_{m}\right) \cdot \boldsymbol{i}_{\text {roll }}\right. \\
& +\left(\cos (\phi) \cdot \boldsymbol{j}_{\text {roll }}-\sin (\phi) \cdot \boldsymbol{k}_{\text {roll }}\right) \cdot \cos \left(\theta_{m}\right) \\
& +\dot{\phi} \cdot \boldsymbol{i}_{\text {roll }} \\
= & \left(\dot{\phi}+\dot{\psi} \cdot \sin \left(\theta_{m}\right)\right) \cdot \boldsymbol{i}_{\text {roll }} \\
& +\dot{\psi} \cdot \cos \left(\theta_{m}\right) \cdot \cos (\phi) \cdot \boldsymbol{j}_{\text {roll }} \\
& -\dot{\psi} \cdot \cos \left(\theta_{m}\right) \cdot \sin (\phi) \cdot \boldsymbol{k}_{\text {roll }} .
\end{aligned}
$$

Equation (23) is then expressed in vector form,

$$
\boldsymbol{w}_{r}^{r}=\left[\begin{array}{c}
\dot{\phi}+\dot{\psi} \cdot \sin \left(\theta_{m}\right) \\
\dot{\psi} \cdot \cos \left(\theta_{m}\right) \cdot \cos (\phi) \\
-\dot{\psi} \cdot \cos \left(\theta_{m}\right) \cdot \sin (\phi)
\end{array}\right] .
$$

The kinetic energy from moment of inertia is

$$
E_{k r I}=\frac{1}{2} \cdot\left(\boldsymbol{w}_{r}^{r}\right)^{T} \cdot \boldsymbol{I}_{r} \cdot\left(\boldsymbol{w}_{r}^{r}\right) .
$$

\section{3) Total kinetic energy:}

The total kinetic energy of roll part is the sum of kinetic . energy from mass center and moment of inertia, and is expressed as

$$
E_{k r}=E_{k r m}+E_{k r I} .
$$

4) Potential energy:

The potential energy of roll part is

$$
E_{p r}=m_{r} \cdot g \cdot \boldsymbol{d}_{o r c}^{b}(2,1) .
$$

where $g$ is acceleration of gravity. $\boldsymbol{d}_{o r c}^{b}(2,1)$ is the second element of $\boldsymbol{d}_{\text {orc }}^{b}$, that is, the element in the direction of $\boldsymbol{j}_{\text {base }}$. 


\section{Pitch part}

1) Kinetic energy of mass center:

The relationship between pitch frame and roll frame is expressed as

$$
\begin{aligned}
\boldsymbol{i}_{\text {pitch }} & =\cos (\theta) \cdot \boldsymbol{i}_{\text {roll }}+\sin (\theta) \cdot \boldsymbol{j}_{\text {roll }} \\
\boldsymbol{j}_{\text {pitch }} & =-\sin (\theta) \cdot \boldsymbol{i}_{\text {roll }}+\cos (\theta) \cdot \boldsymbol{j}_{\text {roll }} \\
\boldsymbol{k}_{\text {pitch }} & =\boldsymbol{k}_{\text {roll }} .
\end{aligned}
$$

From (28) to (30), the rotational matrix from pitch frame to roll frame is obtained

$$
\boldsymbol{R}_{p}^{r}=\left[\begin{array}{ccc}
\cos (\theta) & -\sin (\theta) & 0 \\
\sin (\theta) & \cos (\theta) & 0 \\
0 & 0 & 1
\end{array}\right]
$$

The position vector from $o_{b}$ to mass center of pitch part, expressed in base frame, is

$$
\boldsymbol{d}_{o p c}^{b}=\boldsymbol{d}_{b y}^{b}+\boldsymbol{d}_{y r}^{b}+\boldsymbol{d}_{r p}^{b}+\boldsymbol{d}_{p m c}^{b}
$$

where $\boldsymbol{d}_{r p}^{b}$ is the position vector from $o_{\text {roll }}$ to $o_{\text {pitch }}$, expressed in base frame, and $\boldsymbol{d}_{p m c}^{b}$ is the position vector from $o_{\text {pitch }}$ to mass center of pitch part, expressed in base frame. And they can be obtained by

$$
\begin{aligned}
\boldsymbol{d}_{r p}^{b} & =\boldsymbol{R}_{y}^{b} \cdot \boldsymbol{R}_{r}^{y} \cdot \boldsymbol{d}_{r p}^{r} \\
\boldsymbol{d}_{p m c}^{b} & =\boldsymbol{R}_{y}^{b} \cdot \boldsymbol{R}_{r}^{y} \cdot \boldsymbol{R}_{p}^{r} \cdot \boldsymbol{d}_{p m c}^{p} .
\end{aligned}
$$

The velocity of mass center of pitch part in base frame is

$$
\boldsymbol{v}_{o p c}^{b}=\frac{d\left(\boldsymbol{d}_{o p c}^{b}\right)}{d t}
$$

The kinetic energy of mass center of roll part is

$$
E_{k p m}=\frac{1}{2} \cdot m_{p} \cdot\left\|\boldsymbol{v}_{o p c}^{b}\right\|_{2}^{2} .
$$

\section{2) Kinetic energy of moment of inertia:}

The angular velocity of pitch part expressed in pitch frame is

$$
\boldsymbol{w}_{p}^{p}=\dot{\phi} \cdot \boldsymbol{i}_{\text {roll }}+\dot{\psi} \cdot \boldsymbol{j}_{\text {yaw }}+\dot{\theta} \cdot \boldsymbol{k}_{\text {pitch }} .
$$

Decompose $\boldsymbol{i}_{\text {roll }}, \boldsymbol{j}_{\text {roll }}, \boldsymbol{k}_{\text {roll }}$ into pitch frame.

$$
\begin{aligned}
\boldsymbol{i}_{\text {roll }} & =\cos (\theta) \cdot \boldsymbol{i}_{\text {pitch }}-\sin (\theta) \cdot \boldsymbol{j}_{\text {pitch }} \\
\boldsymbol{j}_{\text {roll }} & =\sin (\theta) \cdot \boldsymbol{i}_{\text {pitch }}+\cos (\theta) \cdot \boldsymbol{j}_{\text {pitch }} \\
\boldsymbol{k}_{\text {roll }} & =\boldsymbol{k}_{\text {pitch }} .
\end{aligned}
$$

Decompose $j_{\text {yaw }}$ in pitch frame,

$$
\begin{aligned}
\boldsymbol{j}_{\text {yaw }}= & \sin \left(\theta_{m}\right) \cdot \boldsymbol{i}_{\text {roll }} \\
& +\cos \left(\theta_{m}\right) \cos (\phi) \cdot \boldsymbol{j}_{\text {roll }} \\
& -\cos \left(\theta_{m}\right) \cdot \sin (\phi) \cdot \boldsymbol{k}_{\text {roll }} \\
= & \sin \left(\theta_{m}\right) \cdot\left(\cos (\theta) \cdot \boldsymbol{i}_{\text {pitch }}-\sin (\theta) \cdot \boldsymbol{j}_{\text {pitch }}\right) \\
& +\cos \left(\theta_{m}\right) \cos (\phi) \cdot\left(\sin (\theta) \cdot \boldsymbol{i}_{\text {pitch }}+\cos (\theta) \cdot \boldsymbol{j}_{\text {pitch }}\right) \\
& -\cos \left(\theta_{m}\right) \cdot \sin (\phi) \cdot \boldsymbol{k}_{\text {roll }} .
\end{aligned}
$$
(42) and $M_{p e}$ are generated by stepper motors. where $M_{y a}$ is the external moment on azimuth axis. $M_{r c}$ is the moment on cross-elevation axis. $M_{p e}$ is the moment

By substituting (38) and (42) into (37),

$$
\begin{aligned}
\boldsymbol{w}_{p}^{p}= & \left(\dot{\phi} \cdot \cos (\theta)+\dot{\psi} \cdot\left(\cos (\theta) \cdot \sin \left(\theta_{m}\right)\right.\right. \\
& \left.\left.+\sin (\theta) \cdot \cos (\phi) \cdot \cos \left(\theta_{m}\right)\right)\right) \cdot \boldsymbol{i}_{\text {pitch }} \\
& +\left(-\dot{\phi} \cdot \sin (\theta)+\dot{\psi} \cdot\left(-\sin (\theta) \cdot \sin \left(\theta_{m}\right)\right.\right. \\
& \left.\left.+\cos (\theta) \cdot \cos (\phi) \cdot \cos \left(\theta_{m}\right)\right)\right) \cdot \boldsymbol{j}_{\text {pitch }} \\
& +\left(-\dot{\psi} \cdot \sin (\phi) \cdot \cos \left(\theta_{m}\right)+\dot{\theta}\right) \cdot \boldsymbol{k}_{\text {pitch }} .
\end{aligned}
$$

Equation (43) can be expressed in vector form by,

$$
\boldsymbol{w}_{p}^{p}=\left[\begin{array}{c}
\dot{\phi} \cdot c \theta+\dot{\psi} \cdot\left(c \theta \cdot s \theta_{m}+s \theta \cdot c \phi \cdot c \theta_{m}\right) \\
-\dot{\phi} \cdot s \theta+\dot{\psi} \cdot\left(-s \theta \cdot s \theta_{m}+c \theta \cdot c \phi \cdot c \theta_{m}\right) \\
-\dot{\psi} \cdot s \phi \cdot c \theta_{m}+\dot{\theta}
\end{array}\right] .
$$

where $c *=\cos (*)$ and $s *=\sin (*)$.

The kinetic energy from moment of inertia is

$$
E_{k p I}=\frac{1}{2} \cdot\left(\boldsymbol{w}_{p}^{p}\right)^{T} \cdot \boldsymbol{I}_{p} \cdot\left(\boldsymbol{w}_{p}^{p}\right) .
$$

3) Total kinetic energy:

The total kinetic energy of pitch part is the sum of kinetic energy from mass center and moment of inertia, and is expressed as

$$
E_{k p}=E_{k p m}+E_{k p I} .
$$

4) Potential energy:

The potential energy of roll part is

$$
E_{p p}=m_{p} \cdot g \cdot \boldsymbol{d}_{o p c}^{b}(2,1)
$$

where $g$ is acceleration of gravity. $\boldsymbol{d}_{o p c}^{b}(2,1)$ is the second element of $\boldsymbol{d}_{\text {opc }}^{b}$, that is, the element in the direction of $\boldsymbol{j}_{\text {base }}$.

\section{Damping energy}

The total damping energy of yaw, roll, and pitch parts are

$$
E_{d}=\frac{1}{2} \cdot C_{d y} \cdot \dot{\psi}^{2}+\frac{1}{2} \cdot C_{d r} \cdot \dot{\phi}^{2}+\frac{1}{2} \cdot C_{d p} \cdot \dot{\theta}^{2} .
$$

E. Lagrange method

The Lagrange equation is

$$
\begin{aligned}
L & =E_{k}-E_{p} \\
& =\left(E_{k y}+E_{k r}+E_{k p}\right)-\left(E_{p y}+E_{p r}+E_{p p}\right) .
\end{aligned}
$$

Apply Lagrange method into yaw, roll, and pitch parts respectively,

$$
\begin{aligned}
& M_{y a}=\frac{d}{d t}\left(\frac{\partial L}{\partial \dot{\psi}}\right)-\frac{\partial L}{\partial \psi}+\frac{\partial E_{d}}{\partial \dot{\psi}} \\
& M_{r c}=\frac{d}{d t}\left(\frac{\partial L}{\partial \dot{\phi}}\right)-\frac{\partial L}{\partial \phi}+\frac{\partial E_{d}}{\partial \dot{\phi}} \\
& M_{p e}=\frac{d}{d t}\left(\frac{\partial L}{\partial \dot{\theta}}\right)-\frac{\partial L}{\partial \theta}+\frac{\partial E_{d}}{\partial \dot{\theta}} .
\end{aligned}
$$


Writing (50) to (52) in the following form

$$
\begin{gathered}
{\left[\begin{array}{c}
M_{y a} \\
M_{r c} \\
M_{p e}
\end{array}\right]=\boldsymbol{M}_{1}\left[\begin{array}{c}
\ddot{\psi} \\
\ddot{\phi} \\
\ddot{\theta}
\end{array}\right]+\boldsymbol{M}_{2}\left[\begin{array}{c}
\dot{\psi}^{2} \\
\dot{\phi}^{2} \\
\dot{\theta}^{2}
\end{array}\right]+\boldsymbol{M}_{3}\left[\begin{array}{c}
\dot{\theta} \cdot \dot{\phi} \\
\dot{\phi} \cdot \dot{\psi} \\
\dot{\psi} \cdot \dot{\theta}
\end{array}\right]} \\
\left.+\boldsymbol{M}_{4}\left[\begin{array}{l}
m_{y} \\
m_{r} \\
m_{p}
\end{array}\right]+\left[\begin{array}{c}
C_{d y} \cdot \dot{\psi} \\
C_{d r} \cdot \dot{\phi} \\
C_{d p} \cdot \dot{\theta}
\end{array}\right]\right)
\end{gathered}
$$

where $M_{1}, M_{2}, M_{3}, M_{4} \in R^{3 \times 3}$.

Isolating acceleration terms will result in

$$
\begin{aligned}
{\left[\begin{array}{c}
\ddot{\psi} \\
\ddot{\phi} \\
\ddot{\theta}
\end{array}\right]=} & -\boldsymbol{M}_{1}^{-1}\left(-\left[\begin{array}{c}
M_{y a} \\
M_{r c} \\
M_{p e}
\end{array}\right]+\boldsymbol{M}_{2}\left[\begin{array}{c}
\dot{\psi}^{2} \\
\dot{\phi}^{2} \\
\dot{\theta}^{2}
\end{array}\right]\right. \\
& \left.+\boldsymbol{M}_{3}\left[\begin{array}{c}
\dot{\theta} \cdot \dot{\phi} \\
\dot{\phi} \cdot \dot{\psi} \\
\dot{\psi} \cdot \dot{\theta}
\end{array}\right]+\boldsymbol{M}_{4}\left[\begin{array}{c}
m_{y} \\
m_{r} \\
m_{p}
\end{array}\right]+\left[\begin{array}{c}
C_{d y} \dot{\psi} \\
C_{d r} \dot{\phi} \\
C_{d p} \dot{\theta}
\end{array}\right]\right) .
\end{aligned}
$$

Euler angles, $[\psi, \phi, \theta]$, are obtained by integrating (54) using Euler method.

\section{Simulation And Validation}

As the scale of complexity of these equations exceeds our handling limit, the practical way in this paper to obtain $\boldsymbol{M}_{1}, \boldsymbol{M}_{2}, \boldsymbol{M}_{3}$ and $\boldsymbol{M}_{4}$ in (53) from (50) to (52), is through the use of Maple software, in which the symbolic calculation is carried out and symbolic results can be converted into Matlab code through one command.

To validate the simulation results of dynamics of MSTA, SimMechanics toolbox is used to generate the reference results. SimMechanics provides a multibody simulation environment for 3D mechanical systems. The mechanical system can be modelled by using blocks representing bodies, joints, constraints, and force elements. SimMechanics formulates and solves the equations of motion for the complete mechanical system [11]. Part of SimMechanics file of MSTA is shown in Figure 2.

The simulation results from (54) using Matlab code are compared with that of SimMechanics, as shown in Figure 3 to Figure 5. The Simulation conditions are:

- $\boldsymbol{M}_{y a}=0, \boldsymbol{M}_{r c}=0$, and $\boldsymbol{M}_{p e}=0$.

- $\psi_{0}=0, \phi_{0}=0, \theta_{0}=0$

where $\psi_{0}, \phi_{0}, \theta_{0}$ are initial values of yaw, roll and pitch. During simulation, the whole mechanical system of MSTA is only acted on by gravity.

From Figure 3 to Figure 5, it can be seen that the simulation results from these two methods are almost the same, which validate the derivation of MSTA dynamic model.

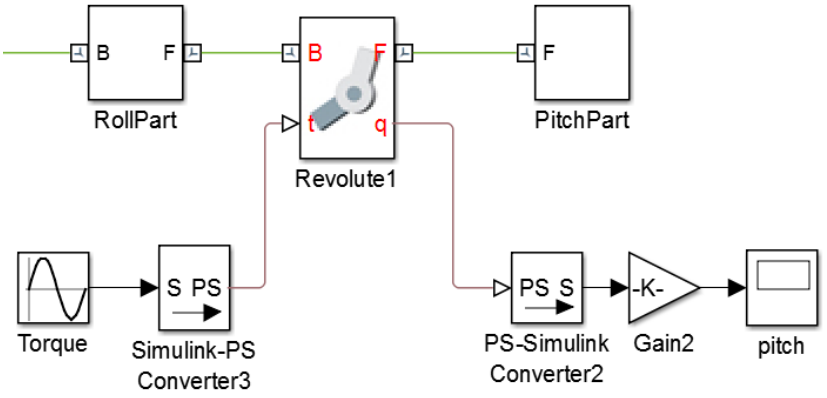

Figure 2. Part of SimMechanics file of MSTA.

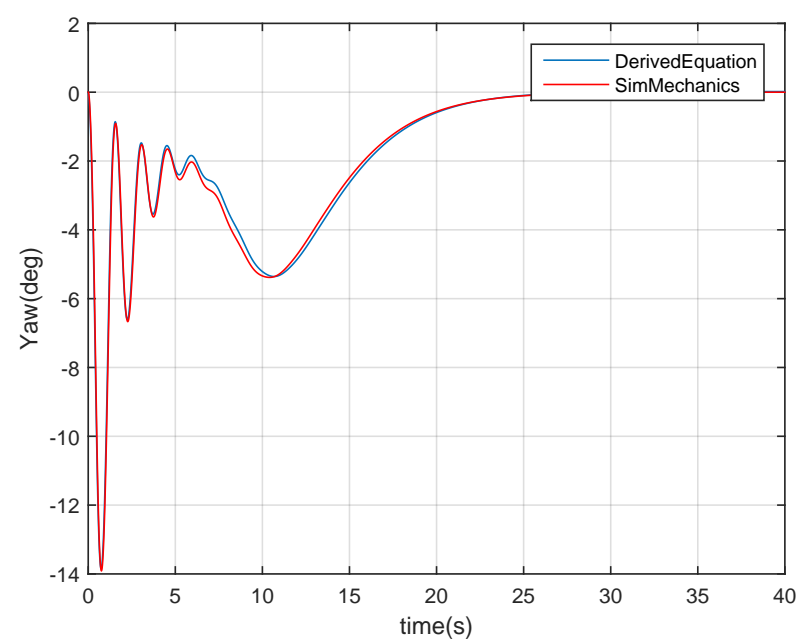

Figure 3. Comparison of yaw from derived equation and SimMechanics.

\section{CONClusion}

The dynamic model of MSTA is firstly derived in this paper using Lagrange method. Instead of using rotational matrix directly, unit vectors of reference frame are used during equation derivation, which can be extended to deal with complicated mechanical systems. Moreover, Maple software is introduced to get coefficient matrices. Finally, the obtained dynamic model is simulated in Matlab using Euler integration method, whose results are compared with that of SimMechanics. From the comparison results, it is seen that the derived dynamic model of MSTA and simulation methods used are correct.

\section{ACKNOWLEDGMENT}

Authors would like to thanks Innovation Fund Denmark for financial support. Thanks also goes to SpaceCom A/S for providing assistance.

\section{REFERENCES}

[1] M. Soltani, R. Izadi-Zamanabadi, and R. Wisniewski, "Reliable control of ship-mounted satellite tracking antenna," Control Systems 


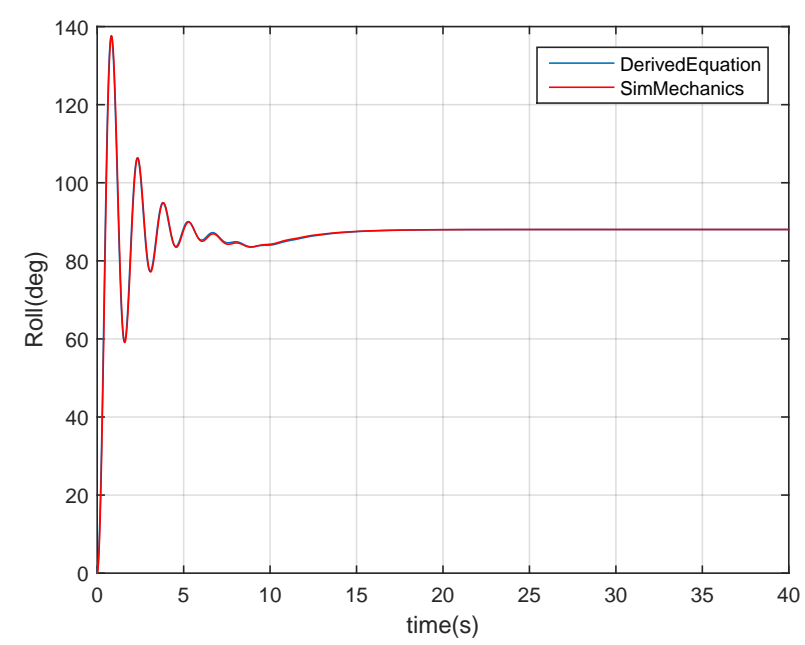

Figure 4. Comparison of roll from derived equation and SimMechanics.

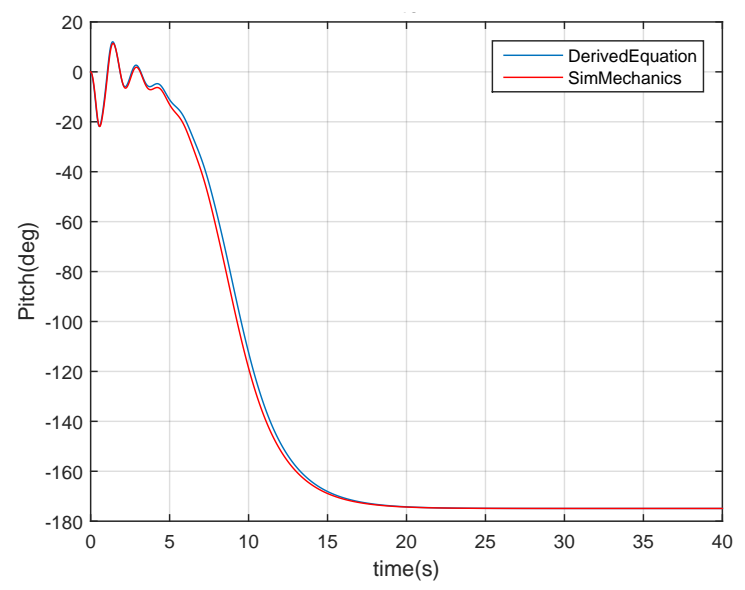

Figure 5. Comparison of pitch from derived equation and SimMechanics.

Technology, IEEE Transactions on, vol. 19, no. 1, pp. 221-228, Jan 2011.

[2] M. Soltani, "Fault diagnosis and fault tolerant control of a shipmounted satellite tracking antenna," PhD thesis, 2008.

[3] S. Soltani, R. Zamanabadi, and R. Wisniewski, "Robust fdi for a shipmounted satellite tracking antenna: A nonlinear approach," in Control Applications, 2008. CCA 2008. IEEE International Conference on, Sept 2008, pp. 757-762.

[4] A. Ming, T. Yamaoka, T. Kida, C. Kanamori, and M. I. Satoh, "Accuracy improvement of ship mounted tracking antenna for satellite communications," in Mechatronics and Automation, 2005 IEEE International Conference, vol. 3, 2005, pp. 1369-1374 Vol. 3.

[5] J. Jiang, J. Guo, B. Yao, and Q. Chen, "Adaptive robust control of mobile satellite communication system with disturbance and model uncertainties," Systems Engineering and Electronics, Journal of, vol. 23, no. 5, pp. 761-767, Oct 2012.

[6] J. Jiang, Q. Chen, B. Yao, and J. Guo, "Desired compensation adaptive robust control of mobile satellite communication system with disturbance and model uncertainties," International Journal of Innovative Computing, Information and Control, vol. 9, no. 1, pp. $153-164,2013$.

[7] S. I. Sagatun and T. Fossen, "Lagrangian formulation of underwater vehicles' dynamics," in Systems, Man, and Cybernetics, 1991. 'De- cision Aiding for Complex Systems, Conference Proceedings., 1991 IEEE International Conference on, Oct 1991, pp. 1029-1034 vol.2.

[8] L. Love, J. F. Jansen, and F. Pin, "On the modeling of robots operating on ships," in Robotics and Automation, 2004. Proceedings. ICRA '04. 2004 IEEE International Conference on, vol. 3, April 2004, pp. 24362443 Vol.3.

[9] H. Pendar, M. Vakil, and H. Zohoor, "Efficient dynamic equations of 3-rps parallel mechanism through lagrange method," in Robotics, Automation and Mechatronics, 2004 IEEE Conference on, vol. 2, Dec 2004, pp. 1152-1157 vol.2.

[10] N. P. TROELS, "Pedestal for tracking antenna," Denmark Patent US2 014299734 (A1), 2014.

[11] Mathworks, "Simmechanics overview." [Online]. Available: http://se.mathworks.com/products/simmechanics/ 